\title{
Study of the Influence of Various Stress-Based Mechanisms on Polarization of an SM mPOF for the Development of Useful Devices
}

\author{
Gaizka Durana, Oskar Arrizabalaga, Eneko Arrospide, Gotzon Aldabaldetreku, Joseba Zubia, and Mikel Azkune
}

\begin{abstract}
In contrast to conventional polymer optical fibres (POFs), single-mode microstructured POFs (SM mPOF) exhibit polarization properties that make them potentially interesting for their use in the design and development of polarimetric fibre optic systems. In spite of the theoretical sixfold symmetry of the microstructure that yields zero linear birefringence, a measurement technique reveals us that the SM mPOF behaves as a linear birefringent system with clearly defined optical axes and characteristics. Regarding externally induced birefringence mechanisms acting upon the SM mPOF, either bend- and pressure-induced retardations or twist-induced light rotation follow the behaviour predicted theoretically. More specifically, bend-induced retardation varies linearly with the inverse square of the bending radius of the fibre, and in the case of asymmetrical lateral stress, pressure-induced retardation varies with the applied force. As to twist-induced rotation, the electric field rotates linearly with the angle through which the fibre is twisted. All cases are highly reproducible phenomena that, if used advantageously, may yield useful mPOF-based polarimetric optical devices not exploited yet.
\end{abstract}

Index Terms-Birefringence, fabrication, optical fiber polarization, optical polymers.

\section{INTRODUCTION}

A LTHOUGH the primary field of application of optical fibres was originally the telecommunications area, their huge potential has made it possible to extend their application to many other working areas. Optical sensing represents one of those fields that relies on the physical aspects of the propagation of light through optical fibres [1]-[3]. Optical sensors based on fibre optics technology are widely used in a broad range of

Manuscript received April 19, 2017; revised May 29, 2017; accepted June 2, 2017. Date of publication June 12, 2017; date of current version June 19, 2017. This work was supported in part by the European Regional Development Fund, in part by the Ministerio de Economía y Competitividad under Project TEC2015-638263-C03-1-R, and in part by the Eusko Jaurlaritza under Project IT933-16, Project ELKARTEK16/45, and Project ELKARTEK16/86. The work of M. Azkune was supported by a research fellowship from the University of the Basque Country (UPV/EHU), Vicerrectorado de Euskara y Plurilingüismo. (Corresponding author: Gaizka Durana).

G. Durana, O. Arrizabalaga, G. Aldabaldetreku, J. Zubia, and M. Azkune are with the Department of Communications Engineering, University of the Basque Country (UPV/EHU), Bilbao E-48013, Spain (e-mail: gaizka. durana@ehu.eus; oskar.arrizabalaga@ehu.eus; gotzon.aldabaldetreku@ehu. eus; joseba.zubia@ehu.eus; mikel.azkune@ehu.eus).

E. Arrospide is with the Department of Applied Mathematics, University of the Basque Country (UPV/EHU), Bilbao E-48013, Spain (e-mail: eneko. arrospide@ehu.eus).

Color versions of one or more of the figures in this paper are available online at http://ieeexplore.ieee.org.

Digital Object Identifier 10.1109/JLT.2017.2713944 applications of science and engineering thanks to their ability to detect many different physical and chemical parameters. Some of them are based on the polarization of light and its sensitivity to environmental changes. In most cases, the fibres used are single-mode to ensure a single wave with single phase and single polarization characteristics.

When polarized light propagates through an optical fibre, both intrinsic and externally induced stress-based mechanisms have to be taken into consideration, since all of them affect to the state of polarization of light. In most cases, fibre birefringence is disturbing and causes unstability problems in the polarization state of the propagating light. In other instances, however, fibre birefringence can be used advantageously for the design and development of useful devices.

Among the various stress-based mechanisms that induce birefringence in optical fibres [4], we will concentrate both on fibre bending and twisting and on asymmetrical lateral stress [5]-[8]. Fibre bending and transverse stress induce linear birefringence, whereas fibre twisting causes circular birefringence in the fibre. In the former case, if linearly polarized light is launched into a fibre, it will become elliptically polarized as it propagates down the fibre. In contrast, in the latter case, linearly polarized light launched into the fibre will rotate in the polarization plane at the output. In the frame of an appropriate experimental configuration, both types of birefringences (linear and circular) may be used for sensing purposes, e.g. pressure sensing, mechanical stress sensing, current sensing, etc. [9]-[12].

Together with the glass optical fibres widely used for longhaul communication links, polymer optical fibres (POFs) have proved themselves useful for a whole range of different sensing applications [13]-[19]. In any case, when reference is made to polarization control in optical fibres, it is implicitly assumed silica as the fibre material; polymer has hardly been regarded as suitable because in POFs the highly multimode nature together with the higher light scattering make them work as light depolarizers, thus preventing their use in such applications. However, with the advent of photonic crystal fibres (PCF) made of polymer [20], [21], it is now possible single-mode guidance in the visible part of the spectrum, paving the way for the use of low cost POFs for the development of highly sensitive optical sensors.

The structure of the paper is as follows. In Section II we start explaining the experimental technique used to systematically determine all the parameters that define the linear birefringent 


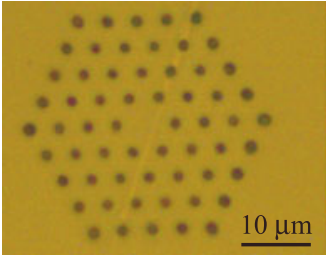

(a)

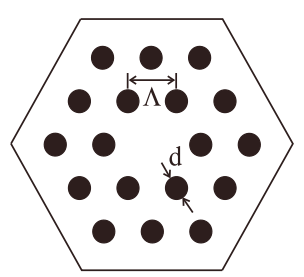

(b)
Fig. 1. (a) Micrograph of the SM mPOF used in the experiments (b) Schematic of the structure with dimensions marked in; $\Lambda$ denotes the distance between neighbouring holes and $d$ the hole diameter.

nature of the fabricated single-mode microstructured POF (SM $\mathrm{mPOF})$. The technique, initially validated on a former commercial mPOF sample [22], has been extensively tested on several SM mPOFs fabricated by us. Then, in Sections III, IV and V, we explain the concept of stress-based birefringence and some of its mechanisms - bend-induced retardation, twist-induced optical activity, and asymmetrical lateral stress, respectively-. We provide experimental results illustrating and confirming them. The measurements are made without having removed the effects of the intrinsic linear birefringence of the fibre. Finally, in Section VI we discuss the results from previous sections and we draw the main conclusions.

\section{MEASUREMENT TEChNIQUE OF INTRINSIC LINEAR BIREFRINGENCE}

Intrinsic birefringence is introduced in optical fibres during the fabrication process. Any effect causing a deviation from the perfect rotational symmetry will contribute to this birefringence. In the case of single-mode fibres, the term birefringence is used in a somewhat different way from its classical meaning; it describes the optical phase difference between the two linearly polarized modes that propagate down the fibre or, in other words, birefringence is present when the degeneracy of the two fundamental modes is lifted.

In order to assure that linear birefringence is the dominant effect on SM mPOFs before applying any external disturbance that may activate external birefringence mechanisms, we made use of a measurement technique to determine, within the laboratory frame of reference, both the spatial orientation of slow and fast axes of the SM mPOF, and the retardation - or mode phase shift $\Delta \varphi$ - induced within the fibre [23].

The geometry of the SM mPOF used is depicted in Fig. 1. It consists of a hexagonal array of small holes defining the cladding region. The fibre was fabricated drilling a monolithic preform of poly(methyl methacrylate) (PMMA) following a four-stage drawing process. In the first stage the primary preform was drawn down to an intermediate cane and then sleeved with a PMMA tube to a diameter of $5.5 \mathrm{~cm}$. Afterwards, the cane was sleeved again with a polycarbonate tube of dimensions $12 / 6$ (12 cm: outer diameter; $6 \mathrm{~cm}$ : inner diameter). Finally, it was drawn down to a fibre of $335 \mu \mathrm{m}$ of diameter. In the final fibre the distance between neighbouring holes was of $\Lambda=5.15 \mu \mathrm{m}$, the hole diameter of $d=2.8 \mu \mathrm{m}$, and the core diameter of $7 \mu \mathrm{m}$. Regarding the thermal pre-treatment of the primary preform, it was annealed in a climatic chamber at $90{ }^{\circ} \mathrm{C}$ for a week in

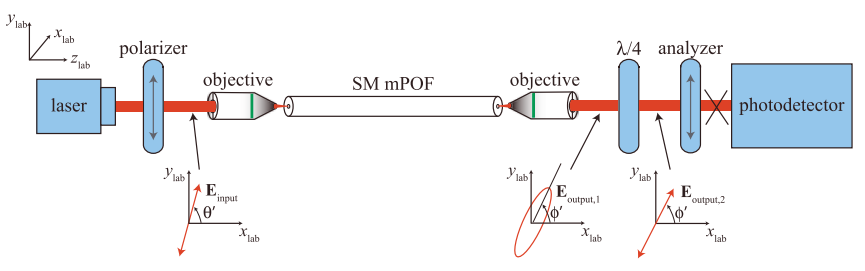

Fig. 2. Experimental set-up used to determine the fast and slow axes, and the retardation of the SM mPOF. The state of polarizations drawn at each stage correspond to a specific system configuration: in this configuration the rotational positions of the quarter-wave retarder and the analyzer are arranged in such a way that no light enters the photodetector.

order to remove any residual stress and humidity. In a similar way, both the cane drawn from the preform and the PMMA and polycarbonate tubes used for sleeving were also annealed at $90^{\circ} \mathrm{C}$ for a week. In any case, the final fibre had no further thermal treatment and, therefore, all the mPOF samples used in the present work were pristine fibres.

The experimental set-up to carry out birefringence measurements is shown in Fig. 2. A linearly polarized light (from a $\mathrm{He}-\mathrm{Ne}$ laser at a wavelength of $632.8 \mathrm{~nm}$ ) making an angle $\theta$ to the slow axis of the fibre - or equivalently, an angle $\theta^{\prime}$ with respect to laboratory axis $x_{\mathrm{lab}}$ - is launched into a short sample of SM mPOF (with a length smaller than $1 \mathrm{~m}$ ) using a microscope objective. The light emerging from the output end of the fibre, elliptically polarized and making an angle $\phi$ between the semi-major axis of the ellipse and the slow axis of the fibre (angle $\phi^{\prime}$ with respect to axis $x_{\text {lab }}$ ), is collimated using an objective microscope and it passes through a quarter-wave plate and a second polarizer before reaching the photodetector. The prime next to an angle denotes that this particular angle has been measured with respect to an arbitrary frame of reference (laboratory frame of reference), whereas those angles without a prime have been measured with respect to the slow axis of the fibre.

The rotational orientation of the fast and slow axes are determined when, firstly, the quarter-wave plate is oriented in such a way that the light emerging from it is linearly polarized, and, secondly, when the light is cancelled at the photodetector by proper orientation of the analyzer. This specific system configuration has been sketched in Fig. 2 by depicting the states of polarization at every stage of the experimental set-up.

At the aforementioned specific configuration of both the quarter-wave plate and the analyzer, if we measure the angle $\xi$ between the slow axis direction of the quarter-wave plate and the polarization axis of the analyzer, then we will be able to determine the absolute orientations of both the input polarizer $(\theta)$ and the quarte-wave plate $(\phi)$-orientations referenced with respect to the slow axis of the fibre - by solving the following system of equations (see the Appendix for further details):

$$
\begin{aligned}
\cos ^{2}(2 \theta)+\cos ^{2} \delta \sin ^{2}(2 \theta) & =\cos ^{2}(2 \xi), \\
\cos \delta \tan (2 \theta) & =\tan (2 \phi),
\end{aligned}
$$

where $\delta$ refers to the linear phase retardance of the fibre after travelling along it. Additionally, the equality $\theta^{\prime}-\phi^{\prime}=\theta-\phi$ holds. 

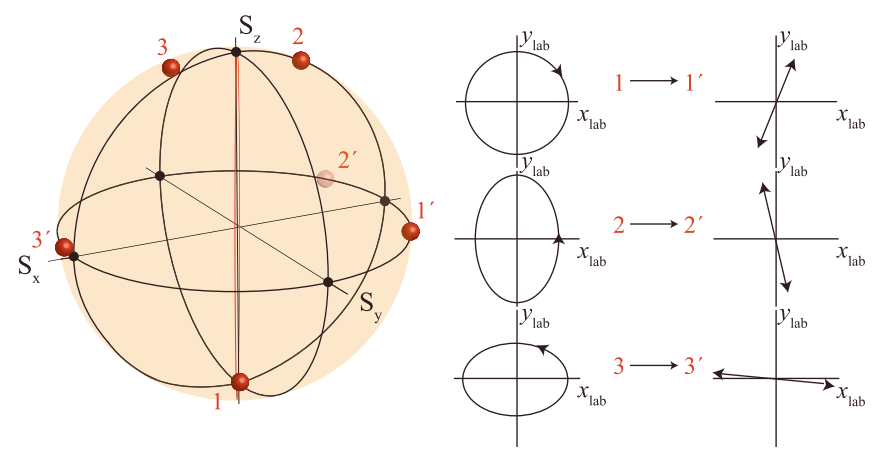

Fig. 3. Measured polarization states of the light emerging from three SM $\mathrm{mPOF}$ samples, and their corresponding position on the surface of the Poincare sphere (polarization states 1,2 and 3). Points $1^{\prime}, 2^{\prime}$ and $3^{\prime}$ on the Poincaré sphere represent linear polarization states, which have been obtained after proper transformation of the corresponding output polarization ( 1,2 and 3 , respectively) by means of rotating the input polarizer an angle $-\theta$, where $\theta$ results from solving the system of equations.

\section{A. Experimental Results}

The experimental results corresponding to three fibre samples obtained from the same drawing process are shown in Fig. 3. The polarization state of the completely polarized light emerging from each SM mPOF sample is characterized by its azimuth $\theta^{\prime}$ and ellipticity $\epsilon$, represented as a single point of longitude $2 \theta^{\prime}$ and latitude $2 \epsilon$ on the surface of the so-called Poincaré Spherea sphere of unit radius used to represent graphically any state of polarization [24] - The polarization of the light emerging from the output end of the fibre was measured using a polarimeter that relies on the rotating wave plate technique. This polarimeter consists of a rotating quarter-wave plate, a linear polarizer and a photodiode.

Let us consider the polarization state 1 from Fig. 3, for which we have determined experimentally $\xi=47.2^{\circ}$ and $\theta-\phi=$ $88.5^{\circ}$. After solving numerically (1), we get $\theta=225^{\circ}$. To check that the optical system is actually behaving as a linear system, the input polarizer is then rotated $-225^{\circ}$ so that $\theta=0^{\circ}$. The resulting output polarization, denoted by $1^{\prime}$ in Fig. 3 , is indeed linearly polarized, as expected for the output light of any linear birefringent system excited with linearly polarized light along any of its optical axes. The same behaviour is observed on the other SM mPOF samples, confirming that, under convenient handling of the fabricated mPOFs, the measurement technique is adequate to assess the predominance of intrinsic linear birefringence in such fibres.

\section{BEND-INDUCED BIREFRINGENCE}

When we bend the fibre, a stress distribution is created inside and, as a consequence, the photoelastic effect produces anisotropic changes of refractive index within the fibre that yields bend-induced linear birefringence, being the fast axis perpendicular to the bending plane [8]. In addition to that, the product of the bend-induced birefringence per unit length of fibre and the square of the bending radius $R$ is constant, i.e., $\beta_{\mathrm{b}} \cdot R^{2}=$ constant, where the constant value depends on geometrical and strain-optical parameters of the optical fibre.

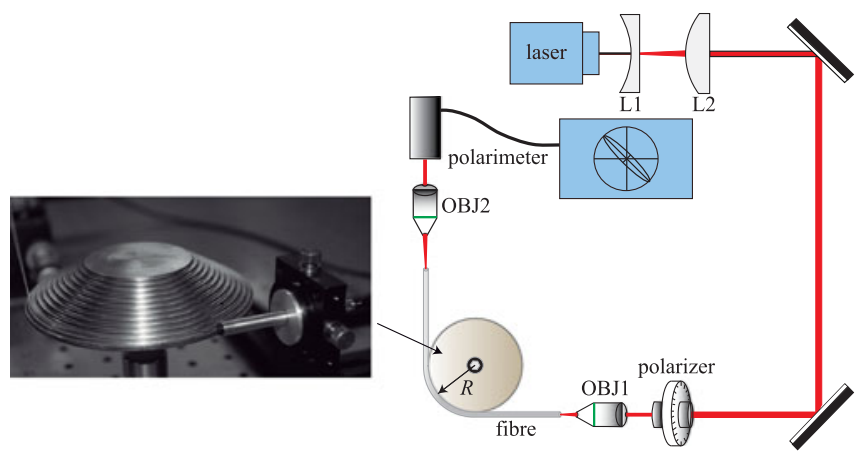

Fig. 4. Schematic of the experimental set-up used to measure bend-induced birefringence. The picture on the left side shows the mechanical configuration used to bend the fibre around cylinders of different radii. L1, L2: lens system for expanding the laser beam; OBJ1, OBJ2: microscope objectives.

\section{A. Experimental Details}

The set-up for launching light into the SM mPOF is shown in Fig. 4. A fibre of length $0.9 \mathrm{~m}(L=0.9 \mathrm{~m})$ is wound a quarter turn around cylinders of different radii ranging from $25 \mathrm{~mm}$ to $50 \mathrm{~mm}$ in steps of $5 \mathrm{~mm}$. Great care has been taken to ensure that the fibre was not twisted or stretched while laying it down. In all the measurements, the polarization axis of the input light has been aligned with the slow axis of the fibre so that in the absence of fibre bending the output polarization is linear. In general, the light emerging from the bent fibre is elliptically polarized as a consequence of induced birefringence. In a similar way as in the previous section, the polarization is measured-the output polarization angle $\phi$, the ellipticity $\epsilon$ and the handedness-using the rotating wave plate technique. Then the retardation or phase difference (in radians) is calculated from the measured results by means of the following equation [25]:

$$
\Delta=\tan ^{-1}(\tan \phi \tan \epsilon)+\tan ^{-1}\left(\frac{\tan \epsilon}{\tan \phi}\right)
$$

where $\Delta$ ranges from $-\pi$ to $\pi$. For linear polarization it takes the values $\Delta=-\pi, 0, \pi$, and for circular polarization $\Delta= \pm \pi / 2$.

\section{B. Experimental Results}

To ensure accurate results, the measurements have been repeated three times on various samples of SM mPOF. Results are shown in Fig. 5, where the average value of the retardation per unit length of bent fibre $\left(\beta_{\mathrm{b}}=2 \Delta / \pi R\right)$ is plotted against the inverse square of the bending radius $(R)$. The vertical bars denote the uncertainty associated with each measurement. As expected from theory, it is found a very clear linear relationship through a Pearson's correlation of 0.99964 . According to the graph, the constant value of $\beta_{\mathrm{b}} \cdot R^{2}$ is equal to $7.0 \times 10^{-3} \mathrm{rad} \cdot \mathrm{m}$. For the low birefrigence fibre used in these experiments the beat length was measured using the fibre cut-back method. The measurements gave a beat length of approximately $12 \mathrm{~cm}$, which results in an intrinsic linear birefringence of $5.3 \times 10^{-6}$ ( $\Delta n \approx 5.3 \times 10^{-6}$ at $\lambda=632.8 \mathrm{~nm}$ ), a value comparable to the linear birefringence induced by a bending radius of approximately $11 \mathrm{~mm}$. 


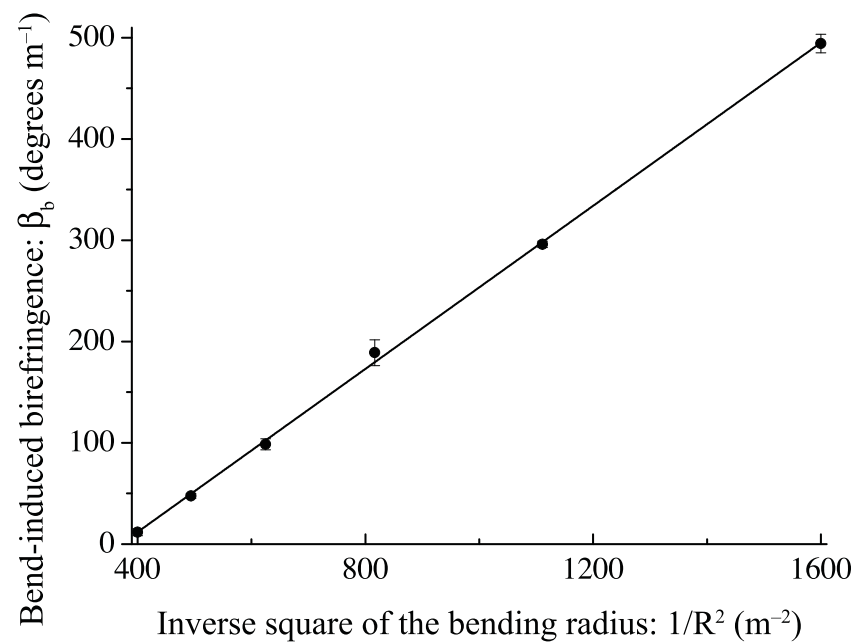

Fig. 5. Graph of bend-induced birefringence as a function of the inverse square of the bending radius. The straight line denotes the best linear fit (Pearson's correlation coefficient: $r=0.99964)$.
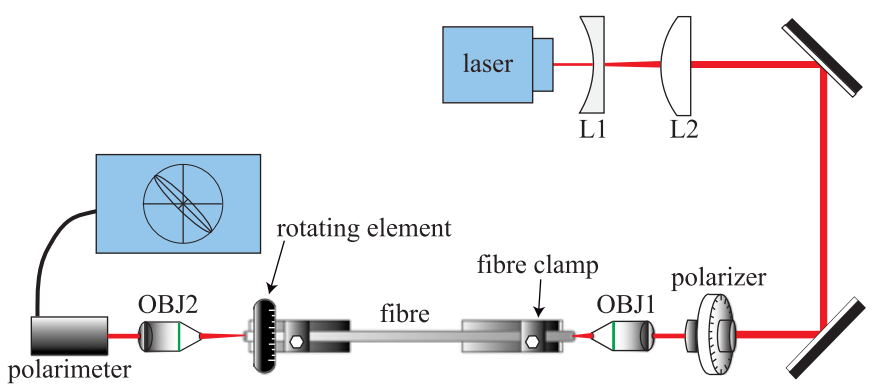

Fig. 6. Schematic of the experimental set-up for twist-induced birefringence measurements. L1, L2: lens system for expanding the laser beam; OBJ1, OBJ2: microscope objectives.

\section{Twist-INDUCED BIREFRINGENCE}

Whenever a fibre is twisted, the shear stress couples the longitudinal field component of one of the modes with the transverse field component of the orthogonal mode, being both components $\pi / 2$ out of phase. This phase difference induces circular birefringence and, as a consequence, a rotation $\alpha$ of the output polarization, which is proportional to the twist angle $\tau$ [6]

$$
\alpha \propto \tau,
$$

where the constant of proportionality depends on the fibre material (its refractive-index and the elements of the strain-optic tensor).

\section{A. Experimental Details}

The schematic of the experimental set-up used to investigate the effect of fibre twisting on the polarization properties of short samples of SM mPOFs is shown in Fig. 6.

Linearly polarized light was launched into short samples of $\mathrm{SM} \mathrm{mPOF}$. One end of the fibre was clampled to an adjustable fibre holder. The other end of the fibre was attached to a rotating element that allowed the fibre to rotate, thus creating fibre torsion. Again, the input linear polarization was aligned with
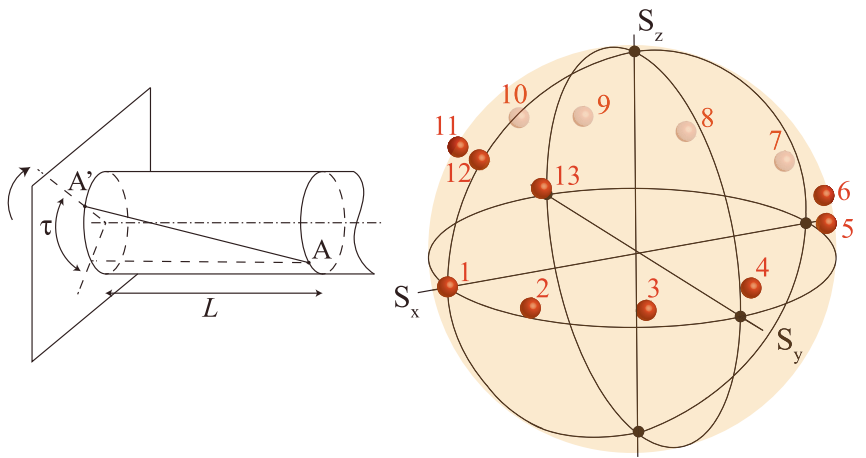

Fig. 7. Evolution of the polarization state on the Poincare sphere as the twist angle is increased in steps of $12^{\circ} .1 \rightarrow$ no twist $(\tau=0) ; 13 \rightarrow \tau=150^{\circ}$. Fibre length: $L=0.9 \mathrm{~m}$.

the slow axis of the fibre so that in the absence of any fibre torsion the polarization of the output light was linearly polarized and parallel to the input linear polarization. Likewise, great care was taken to minimize the effects of clamping pressure and fibre manipulation on the output polarization. Therefore, no extra retardation was induced in the fibre as a consequence of undesirable induced birefringences, i.e., photoelastic effects.

\section{B. Experimental Results}

The pictorial representation of Fig. 7 shows the effect of torsion on the state of polarization or, more specifically, the influence of several potentially coexisting birefringences on the evolution of the state of polarization of the output light.

For a fibre of length $L=0.9 \mathrm{~m}$ with pure circular birefringence $\beta_{\mathrm{t}}$, the state of polarization would rotate on the surface of the sphere around the polar axis through an angle $\beta_{\mathrm{t}} L$ (rotation along the equator). However, the experimental results reveal that the state of polarization starts at equatorial point 1 (linear polarization) and evolves following a helix-like path, thus deviating progressively from that equatorial path due to an increase in light ellipticity with applied fibre torsion. Nevertheless, it should be noted that, in terms of path deviation from that expected for pure circular birefringence, the experimental data from Fig. 7 correspond to the worst experimental case; we observed that for other SM mPOF samples of shorter length, the ellipticity did hardly increase with fibre torsion. The probable reason of such an increase in polarization ellipticity may be found on the coupled birefringences arisen during fibre set-up.

The same experimental data set is also shown in Fig. 8, but broken down into two graphs, one showing the evolution of the azimuth $\phi$ with fibre torsion (Fig. 8, top), and the other one plotting the ellipticity $\epsilon$ as a function of fibre torsion (Fig. 8, bottom). Again, each set of experimental data has been repeated three times, and the average value and the corresponding standard deviation have been plotted. The polarization rotation angle $\alpha$ of the output polarization with respect to that in which the fibre lies down flat without any torsion is linearly related to the twist angle $\tau$ and quite independent of the fibre length. The ratio $\alpha / \tau$ has been found to be around 1.3, although some variability has also been observed, which is most probably related to how 

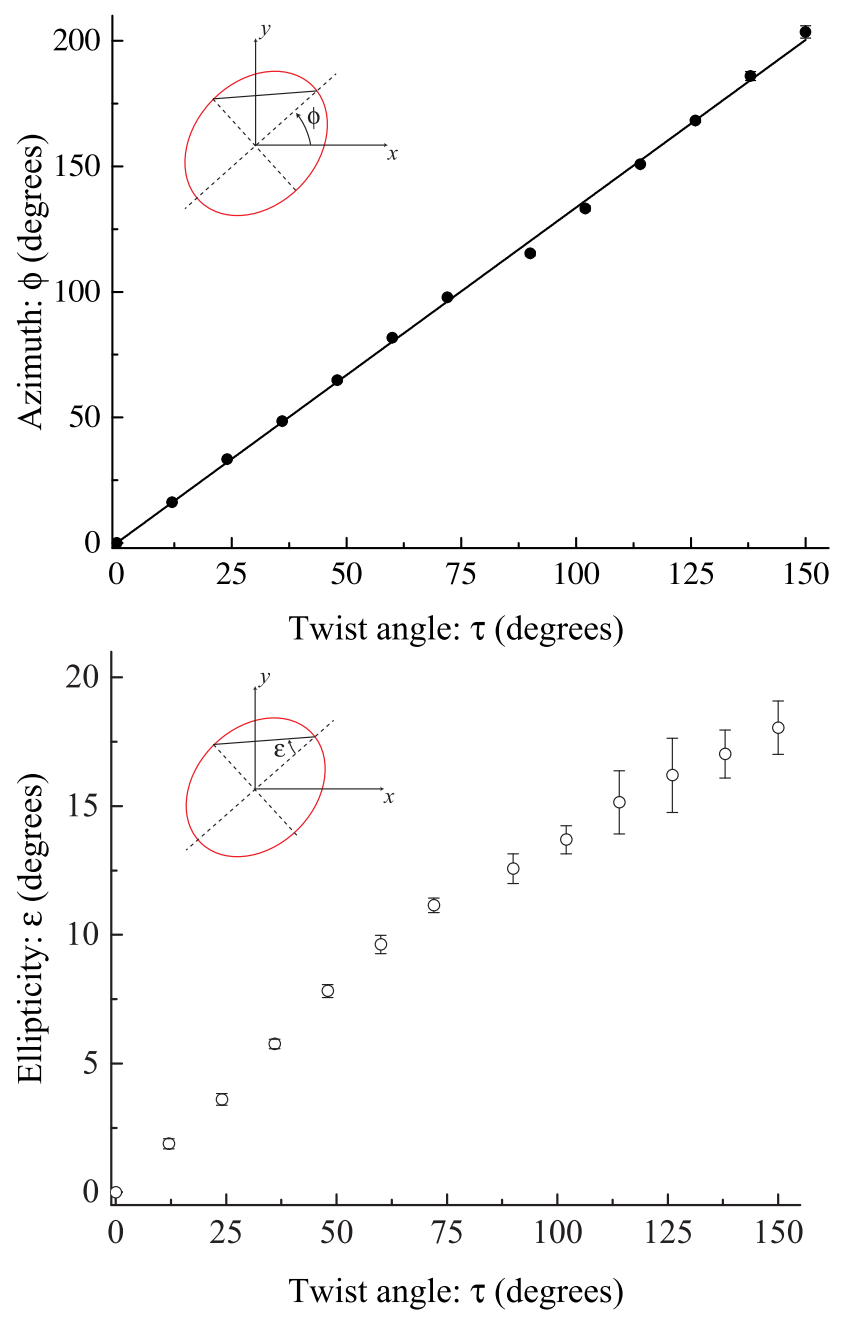

Fig. 8. Graph of the azimuth (upper graph) and the ellipticity (lower graph) as a function of the twist angle. The straight line on the upper graph denotes the best linear fit (Pearson's correlation coefficient: $r=0.99952)$. Fibre length: $L=0.9 \mathrm{~m}$. The input linearly polarized light is parallel to the slow axis of the $\mathrm{SM} \mathrm{mPOF}$.

taut the fibre was stretched when laying it down flat without any torsion $(\tau=0)$.

The Pearson's correlation coefficient of 0.99952 confirms the high linearity of the polarization rotation.

\section{TRANSVERSE STRESS}

When the fibre is pressed into an angled V-groove, linear birefringence is induced [6]. In this case, the induced photoelastic birefringence is proportional to the applied force $f$ :

$$
\beta \propto(1-\cos 2 \delta \sin \delta) \frac{f}{\pi r E},
$$

where $\beta=k_{0}\left(n_{x}-n_{y}\right)$ is the phase difference between the two propagating modes with perpendicular polarizations, $f$ the linear force per unit length, $r$ the outer radius of the fibre, $E$ Young's modulus of the fibre material, and $2 \delta$ the angle of the V-groove. Fig. 9 shows the geometry of the external birefringence mechanism.

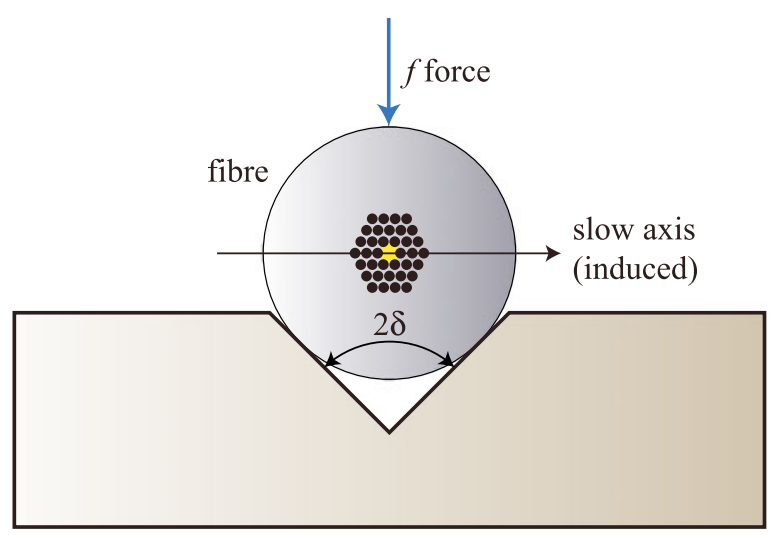

Fig. 9. External birefringence mechanism induced by an external force action $f$.

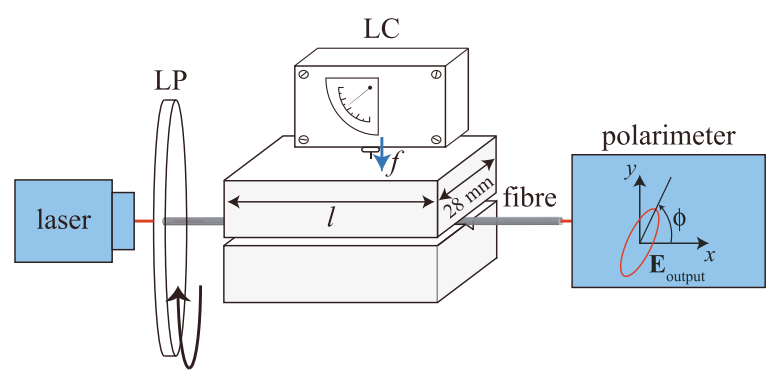

Fig. 10. Schematic of the experimental set-up used to determine the influence of applied external force on light polarization at the fibre output. LP: linear polarizer, LC: load cell.

\section{A. Experimental Details}

As shown in Fig. 10, the experimental set-up consists of a laser, a rotating linear polarizer, a load cell to apply a certain amount of localized force $f$ to a SM mPOF pressed into a V-groove, and the same polarimeter used in Sections II, III, and IV. The light at the fibre input is linearly polarized along the slow axis of the SM mPOF so that in the absence of any applied force the output light remains linearly polarized along the same axis. The other end of the fibre is connected to the polarimeter in order to analyse the state of polarization of the output light. Then the phase difference is calculated by means of (2).

The force $f$ is applied to the fibre by means of a metallic cylindric tip with a flat end surface (diameter: $0.85 \mathrm{~mm}$ ) that presses a piece made of PMMA with a flat surface (width: $28 \mathrm{~mm}$, length: $l$ ).

All measurements have been carried out in the following way: first of all, the SM mPOF sample is laid onto the V-groove and the PMMA piece is placed on top of it carefully to avoid inducing any extra retardation. Thus, the linear polarization parallel to the fibre slow axis is maintained at the fibre output. On each measurement, a load is applied, the state of polarization is measured and then the load is removed until the state of polarization recovers its original condition - linear and parallel to the fibre slow axis- Then, the load is increased in steps of $0.5 \mathrm{~N}$ and the described measurement process is repeated. 


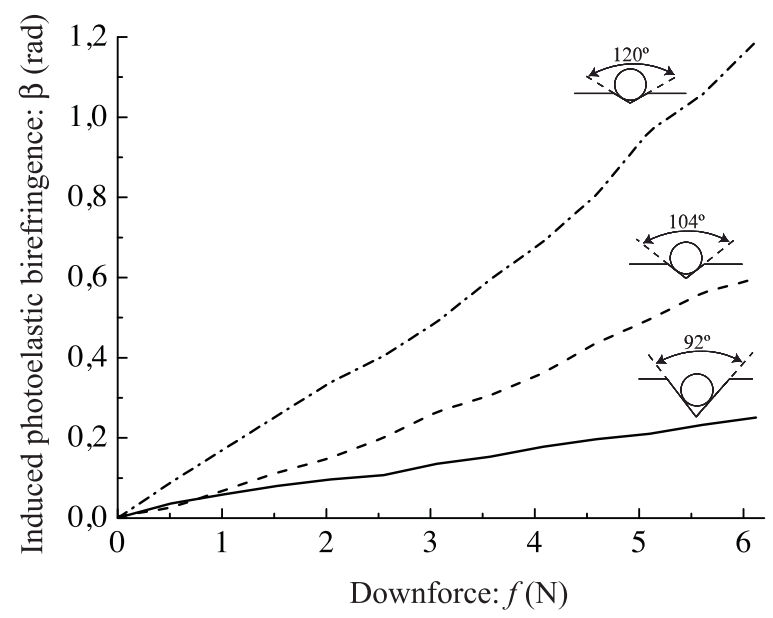

Fig. 11. Induced phase difference (photoelastic birefringence) as a function of the applied external downforce for different V-groove angles.

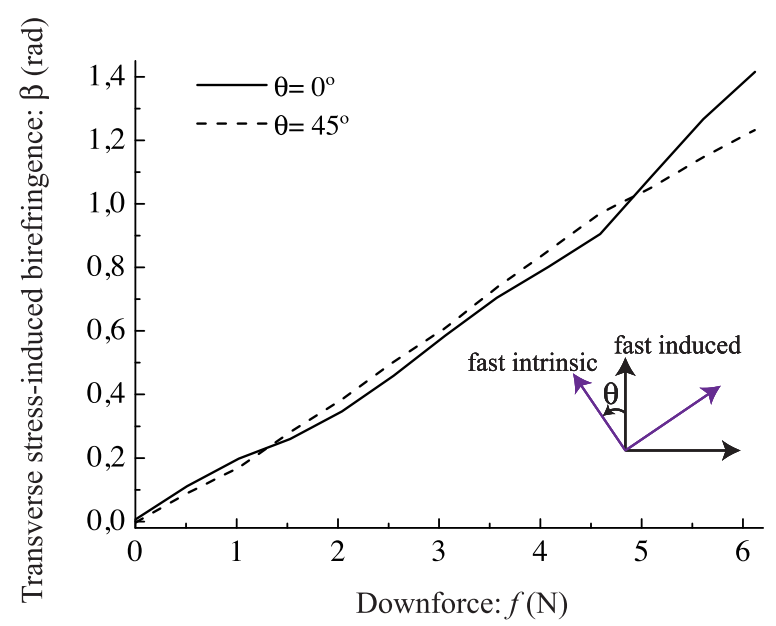

Fig. 12. Transverse stress-induced birefringence on the SM mPOF as a function of the applied external downforce for two different rotational orientations of the fibre. The angle $\theta$ refers to the angle between the fibre intrinsic fast axis and the vertical direction (induced fast axis). V-groove angle: $2 \delta=120^{\circ}$.

\section{B. Experimental Results}

Fig. 11 shows the birefringence induced in the SM mPOF lying on different $\mathrm{V}$-grooves of angle $2 \delta$ under the influence of the external downforce $f$. As expected from (4), for a fixed value of the applied force $f$, the induced birefringence increases with the $\mathrm{V}$-groove angle $2 \delta$, and for a fixed value of the $\mathrm{V}$-groove angle $2 \delta$, the phase difference increases quite in a linear fashion with the applied force $f$. We have observed that the contribution to such an increase comes mainly from the polarization ellipticity $\epsilon$, whereas the azimuth $\phi$ remains almost constant.

We have also checked the possible effects of the rotational orientation of the fibre lying on the $\mathrm{V}$-groove. For such a purpose, we have considered a $\mathrm{V}$-groove of angle $2 \delta=120^{\circ}$. The curves shown in Fig. 12 correspond to the case in which the intrinsic and induced fast axes coincide $\left(\theta=0^{\circ}\right.$, solid line), and to the case in which the angle between them is of $45^{\circ}\left(\theta=45^{\circ}\right.$, dashed line). The phase difference seems to be quite independent of the rotational position of the fibre.

\section{DISCUSSION AND CONCLUSION}

In contrast to conventional POFs, SM mPOFs adapt well to polarization experiments. Although an ideal PCF with a structure of six-fold symmetric has zero linear birefringence, the fibre measurement method reports a well-defined linear behaviour of the fibre as a consequence of the fabrication process that creates a low birefringence fibre. The fibre shows an intrinsic linear birefringence of the order of $5.3 \times 10^{-6}$ at $\lambda=632.8 \mathrm{~nm}$.

The relative phases of both polarization modes are very sensitive to external perturbations. In the $\mathrm{SM}$ mPOFs fabricated by us, the birefringence induced in the fibre as a consequence of fibre bending, twisting and/or asymmetrical lateral stress match well the predictions made by theoretical analyses. In the case of fibre bending and asymmetrical lateral stress, the induced phase difference (or retardation) changes linearly with the inverse square of the bending radius and the applied external force, respectively. It is worthy of mention that our analysis is restricted to a fixed value of the design parameter $d / \Lambda$ of the $\mathrm{mPOF}$, since a change in the hole diameter might also modify the stressinduced linear and circular birefringences. This assumption is based on the results obtained for glass microstructured optical fibres in [26], results that may be extrapolated reasonably well to the case of mPOFs. Even though the study of the dependence of stress-induced birefringences on the design parameter $d / \Lambda$ of the mPOF would be of great interest, such a study is beyond the scope of the present work.

The fact of fixing the fibre with a clamp induces additional birefringence in the fibre that may affect the state of polarization of the output light. This point is particularly important to keep in mind in applications that require birefringence-free clamping of the fibre. Birefringence-free holding is not possible when fixing the fibre with a V-groove, having to take into account that the Vgroove angle also affects to the degree of induced birefringence. Indeed, the induced birefringence in the fibre increases with the V-groove angle. This birefringence mechanism may also be useful for the detection of some physical parameters that induce this type of birefringence in the fibre.

Finally, the ratio of the twist-induced polarization rotation $\alpha$ to the twist angle $\tau$, i.e., the ratio $\alpha / \tau$, is of approximately 1.3 , in contrast to the experimental value of 0.069 published for glass single-mode optical fibres [5]. Therefore, a $90^{\circ}$ twist in the $\mathrm{SM}$ mPOF produces a rotation of approximately $120^{\circ}$ in the direction of linear polarization.

\section{APPENDIX}

Let us consider the optical fibre as a birefringent system with linear phase retardance $\delta$. If linearly polarized light is launched at an angle $\theta$ with respect to the slow axis ( $x$-axis), the light will emerge elliptically polarized with the semi-major axis making an angle $\phi$ to the slow axis. Using Jones calculus, the electric field at the fibre output may be written as

$$
\left[\begin{array}{l}
E_{x} \\
E_{y}
\end{array}\right]=\left[\begin{array}{cc}
\exp (\jmath \delta) & 0 \\
0 & 1
\end{array}\right] \times\left[\begin{array}{c}
\cos \theta \\
\sin \theta
\end{array}\right] E_{0} \exp (\jmath \omega t),
$$




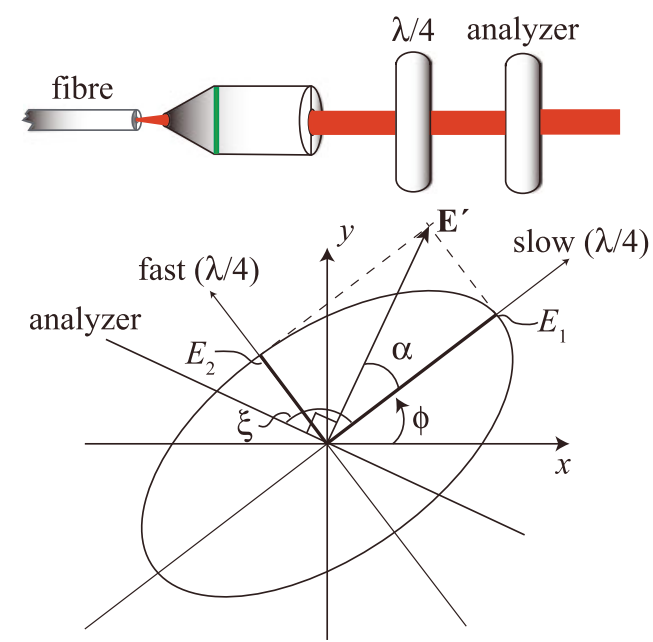

Fig. 13. Coordinate system used in the calculations.

or, if $E_{1}$ and $E_{2}$ represent the electric field along the semi-major and semi-minor axes, respectively, then

$$
\left[\begin{array}{l}
E_{1} \\
E_{2}
\end{array}\right]=\left[\begin{array}{cc}
\cos \phi & \sin \phi \\
-\sin \phi & \cos \phi
\end{array}\right] \times\left[\begin{array}{c}
E_{x} \\
E_{y}
\end{array}\right]
$$

The polarization parameter $P$, defined as

$$
P=\frac{\left|E_{1}\right|^{2}-\left|E_{2}\right|^{2}}{\left|E_{1}\right|^{2}+\left|E_{2}\right|^{2}}
$$

measures the ellipticity of the output light. If we combine (5), (6) and (7), and after suitable manipulation, it may be shown that

$$
P^{2}=\cos ^{2}(2 \theta)+\cos ^{2}(\delta) \sin ^{2}(2 \theta)
$$

and

$$
\tan (2 \phi)=\cos \delta \tan (2 \theta) .
$$

If we now place a quarter-wave plate at the exit of the fibre oriented in such a way that the light emerging from the plate is linearly polarized (electric field $\mathbf{E}^{\prime}$ in Fig. 13; the semi-major and semi-minor axes of the polarization ellipse are parallel to the slow and fast axes of the retarder), this light may be cancelled with a properly oriented polarizer (the analyzer in Fig. 13). At this specific configuration, and making use of (7) and the trigonometric expressions that relate $\alpha, E_{1}$, and $E_{2}$, the polarization parameter becomes

$$
P^{2}=\cos ^{2}(2 \alpha) .
$$

Finally, the relationship $\xi-\alpha=\pi / 2$ yields $P=\cos (2 \xi)$, where $\xi$ is the angle between the fast-axis of the quarter-wave plate and the analyzer.

Therefore, by measuring experimentally the angle $\xi, P$ may be found, and the relative angle $\theta-\phi$ between the input polarizer and the fast axis of the quarter-wave plate may also be determined. Additionally, by solving (8) and (9) we can determine the absolute values of $\theta$ and $\phi$.

\section{REFERENCES}

[1] W. Lin, C. Zhang, L. Li, and S. Liang, "Review on development and applications of fiber-optic sensors," in Proc. IEEE Symp. Photon. Optoelectron., 2012.

[2] K. Peters, "Polymer optical fiber sensors-A review," Smart Mater. Struct., vol. 20, pp. 1-17, 2010.

[3] J. M. Lopez-Higuera, Handbook of Optical Fibre Sensing Technology. West Sussex, U.K.: Wiley, 2002.

[4] S. C. Rashleigh, "Origins and control of polarization effects in singlemode fibers," J. Lightw. Technol., vol. LT-1, pp. 312-331, Jun. 1983.

[5] A. M. Smith, "Birefringence induced by bends and twists in single-mode optical fiber," Appl. Opt., vol. 19, pp. 2606-2611, 1980.

[6] R. Ulrich and A. Simon, "Polarization optics of twisted single-mode fibers," Appl. Opt., vol. 18, pp. 2241-2251, 1979.

[7] A. Kumar and R. Ulrich, "Birefringence of optical fiber pressed into a V-groove," Opt. Lett., vol. 6, pp. 644-646, 1981.

[8] R. Ulrich, S. C. Rashleigh, and W. Eickhoff, "Bending-induced birefringence in single-mode fibers," Opt. Lett., vol. 5, pp. 273-275, 1980.

[9] D. Chardon and S. J. Huard, "A new interferometric and polarimetric temperature optical fiber sensor," J. Lightw. Technol., vol. LT-4, pp. 720-725, Jul. 1986.

[10] W. J. Bock, T. R. Wolinski, and A. Eftimov, "Polarimetric fibre-optic strain gauge using two-mode highly birefringent fibres," Pure Appl. Opt., vol. 5, pp. 125-139, 1996.

[11] J. Calero, S.-P. Wu, C. Pope, S. L. Chuang, and J. P. Murtha, "Theory and experiments on birefringent optical fibers embedded in concrete structures," J. Lightw. Technol., vol. 12, pp. 1081-1091, Jul. 1994.

[12] A. Anuszkiewicz, T. Martynkien, J. Olszewski, P. Mergo, and W. Urbanczyk, "Polarimetric sensitivity to hydrostatic pressure and temperature in a side-hole fiber with squeezed microstructure," J. Opt., vol. 17, 2015, Art. no. 125609

[13] J. Zubia and J. Arrue, "Plastic optical fibers: An introduction to their technological processes and applications," Opt. Fiber Technol., vol. 7, pp. 101-140, 2001.

[14] O. Ziemann, J. Krauser, P. E. Zamzow, and W. Daum, POF Handbook: Optical Short Range Transmission Systems. Berlin, Germany: Springer, 2008.

[15] P. Stajanca, O. Cetinkaya, M. Schukar, P. Mergo, D. J. Webb, and K. Krebber, "Molecular alignment relaxation in polymer optical fibers for sensing applications," Opt. Fiber Technol., vol. 28, pp. 11-17, 2016.

[16] Y. Luo, B. Yan, Q. Zhang, G.-D. Peng, J. Wen, and J. Zhang, "Fabrication of polymer optical fibre (POF) gratings," Sensors, vol. 17, p. 511, 2017.

[17] C. A. F. Marques, P. Antunes, P. Mergo, D. J. Webb, and P. Andre, "Chirped Bragg gratings in PMMA step-index polymer optical fiber," IEEE Photon. Technol. Lett., vol. 29, pp. 500-503, Mar. 2017.

[18] D. J. Webb, "Fibre Bragg grating sensors in polymer optical fibres," Meas. Sci. Technol., vol. 26, 2015, Art. no. 092004.

[19] X. Hu et al., "Polarization effects in polymer FBGs: Study and use for transverse force sensing," Opt. Express, vol. 23, pp. 4581-4590, 2015.

[20] M. A. van Eijkelenborg, "Microstructured polymer optical fibre," Opt. Express, vol. 9, pp. 319-327, 2001.

[21] M. Large, L. Poladian, G. Barton, and M. A. van Eijkelenborg, Microstructured Polymer Optical Fibres. New York, NY, USA: Springer, 2007.

[22] G. Durana, O. Arrizabalaga, G. Aldabaldetreku, M. A. Illarramendi, and J. Zubia, "Stress-based mechanisms in polymer mPOFs for fibre optic sensing," Proc. SPIE, vol. 9157, 2014, Art. no. 91572J.

[23] A. M. Smith, "Automated birefringence measurement system," J. Phys. E: Sci. Instrum., vol. 12, pp. 927-930, 1979.

[24] D. H. Goldstein, Polarized Light. Boca Raton, FL, USA: CRC Press, 2003.

[25] M. Born and E. Wolf, Principles of Optics. London, U.K.: Cambridge Univ. Press, 1999.

[26] Z. Zhu and T. G. Brown, "Stress-induced birefringence in microstructured optical fibers," Opt. Lett., vol. 28, pp. 2306-2308, 2003.

Authors' biographies not available at the time of publication. 